\title{
Aberration correction in electron microscopy and spectroscopy
}

\author{
Ondrej L. Krivanek ${ }^{1,2}$
}

${ }^{1}$ Nion R\&D, 11515 NE 118th St., Kirkland, WA 98034, USA, ${ }^{2}$ Department of Physics, Arizona State University, Tempe, AZ 85287, USA

Aberration correction in electron microscopy has its origins in the work of Scherzer [1], who showed in 1936 that aberrations of round electron lenses are unavoidable and limit the attainable spatial resolution. In 1947, Scherzer went on to propose several approaches to counter the aberrations [2], and his proposals were followed by a period of design and building of aberration correctors. New correction principles were discovered and there were several successful 'proof of principle' correctors built, but the first successful correctors that improved the resolution of the microscopes they were built into were only introduced a half century later, in the late 1990s [3-6] (see [7] for a thorough review). The long wait for eventual success made many people think that practical aberration correction may never work out.

Fig. 1 documents the spatial resolution improvement made possible by aberration correction. Instead of showing 1/resolution, as was done previously $[8,9]$, the graph shows the progress in a figure of merit (FOM) defined as the electron wavelength $\lambda$ divided by the spatial resolution $\mathrm{d}$. The FOM corresponds to the angular range used for image formation (f-stop in camera parlance), and it has the advantage that it remains nearly constant for microscopes using the same type of technologies but different accelerating voltages. The blue diamonds track the progress of non-corrected transmission electron microscopes (TEMs) and show that the TEMs reached a "ceiling" of $\lambda / \mathrm{d} \sim 0.01$ in the nineteen seventies for $100 \mathrm{kV}$ microscopes, eighties for $300-400 \mathrm{kV}$ ones, and late nineties for high voltage instruments $(\geq 1 \mathrm{MV})$. There were, however, three notable exceptions: Crewe and Wall's annular dark field (ADF) scanning TEM (STEM) imaging increased $\lambda / \mathrm{d}$ to around $0.024(\mathrm{~d} / \lambda=42)$ already in 1975; Nellist, McCallum and Rodenberg's first resolution-improving ptychographic reconstruction increased it to $0.027(\mathrm{~d} / \lambda=37)$ in 1995; and O'Keefe et al.'s TEM image processing using through-focus reconstruction reached $0.022(\mathrm{~d} / \lambda$ $=45$ ) in 2001. However, reconstruction methods cannot increase the spatial resolution of microanalysis, and the prospects that the ceiling would be completely shattered without new technologies coming into play were essentially nil.

Correcting the spherical aberration of TEMs (red squares) increased $\lambda / \mathrm{d}$ to around 0.018 at first, and subsequent developments in corrector technologies have brought it to $0.07(\mathrm{~d} / \lambda=15)$. The hardware progress therefore amounts to increasing $\lambda / \mathrm{d}$ by $\sim 3000 \mathrm{x}$ relative to the first electron microscope. Combining aberration correction with ptychographic processing has increased the FOM further to 0.1 (d/ $\lambda$ $=10)$ [10]. Continued future progress is likely, as there is no ceiling we are currently pushing against, but light-optical performance levels of $\lambda / \mathrm{d} \geq 1$ are likely to remain out of reach. Fortunately, there are few structures in our world, made of atoms $1 \AA$ or further apart, that cannot be resolved with $0.5 \AA$ or even 1 $\AA$ resolutions, and at $\lambda / \mathrm{d}=0.1$, these levels are reached at 57 and $15 \mathrm{kV}$ primary voltage, respectively.

The improved resolution has led to many new capabilities in electron microscopy. Some of them were easy to foresee, such as spatial resolution improvement by 2-3x (and more with more sophisticated corrector designs), improved sensitivity to light elements, and $>10 x$ increase in the available probe current in the STEM. Other advances were less obvious, at least in the beginning, e.g. aberration correction allowing atomic resolution to be reached at lower operating voltages and thus avoiding knock-on damage 
in light $\mathrm{Z}$ materials, with a large impact on the study of 2D materials, their defects and transformations; and a substantial improvement in the resolution attainable in the beam direction. The less-foreseeable progress also includes advances in related fields such as ptychography and 4D STEM in general.

Aberration correction has led to another major benefit in electron microscopy, not always fully appreciated: it has allowed the energy resolution of electron energy-loss spectroscopy (EELS) carried out in the (S)TEM to reach unprecedented levels. Fig. 2 documents the progress in this field, using an FOM equal to $1 /(\delta \mathrm{E} \lambda)$, where $\delta \mathrm{E}$ is the energy resolution. Reaching good energy resolution is much easier in systems that operate at lower primary voltages, and sub-meV resolution is relatively straightforward in broad-beam systems operating at just a few V [11]. Compensating for this trend with an FOM provides a better perspective than looking at the energy resolution itself. Our practical experience shows that the EELS resolution of a particular instrument operating at a chosen primary voltage is proportional to $\lambda^{-1}$, as expected since $\delta \mathrm{E}$ in our ground-potential system is given by [12]: $\boldsymbol{\delta} \boldsymbol{E}=\boldsymbol{d}_{\boldsymbol{S E \boldsymbol { P }}} / \boldsymbol{\kappa}$, where $\boldsymbol{d}_{\boldsymbol{S E \boldsymbol { P }} \boldsymbol{P}}$ is the physical size of a single-energy peak on the EELS detector (and on the monochromator energy-selecting slit) and $\boldsymbol{\kappa}$ the energy dispersion (usually measured in $\mu \mathrm{m} / \mathrm{eV}$ ), and further $\boldsymbol{d}_{\boldsymbol{S E} \boldsymbol{P}} \alpha \lambda$ and $\boldsymbol{\kappa} \alpha \square \square \square \square \alpha \lambda^{2}$, giving $\delta \boldsymbol{E} \propto \lambda^{-1}$ (for non-relativistic voltages of primary interest here).

An FOM equal to $1 /(\delta \mathrm{E} \lambda)$ therefore gives a single point for each EELS system, rather than a collection of points for different values of $\mathrm{V}_{\mathrm{o}}$. Unlike the FOM used here for spatial resolution, the energy resolution FOM is unfortunately not dimension-less, and its magnitude therefore depends on the chosen units - meV and $\AA$ in our case. There are other caveats concerning the applicability of the FOM to EELS systems limited by the energy spread of the source and by system instabilities, and also to systems using decelerating-accelerating monochromators and spectrometers. But it applies accurately to groundpotential monochromated EELS, and it appears to be a reasonable compromise for the other systems.

Similar to the progress in spatial resolution, the resolution of non-monochromated EELS systems reached a ceiling at $1 /(\delta \mathrm{E} \lambda) \sim 0.1$, for EELS systems spanning from $100 \mathrm{kV}$ to $1 \mathrm{MV}$. Monochromators allowed the resolution to progress further, and the latest systems, which employ extensive aberration correction in both the monochromator and the spectrometer, as well as novel stabilization schemes [12, 13], have increased $1 /(\delta \mathrm{E} \lambda)$ to 3.4 - about $3000 \mathrm{x}$ increase relative to the first EELS instruments, i.e. similar to the advance in the spatial resolution FOM.

Table 1 shows how the FOM values translate into attainable energy resolutions at different primary voltages. 20-30 meV energy resolution opened up the new field of vibrational spectroscopy in the electron microscope $[14,15]$, and subsequent progress to $5 \mathrm{meV}$ resolution and below has made the technique more versatile and powerful. Figs 3 and 4 illustrate two of the more remarkable recent results: detecting the substitution of ${ }^{12} \mathrm{C}$ by ${ }^{13} \mathrm{C}$ by monitoring a $4.8 \mathrm{meV}$ energy shift of a vibration peak due to a $\mathrm{C}-\mathrm{O}$ stretch [16]; and detecting the vibrational signature of a single Si atom impurity in graphene [17]. Other breakthrough experiments have analyzed atomic-scale variations in the vibrational signal of crystalline $\mathrm{Si}$ [18], determined the local temperature of a sample from the ratio of energy gains to energy losses [19, 20], detected hydrogen and analyzed its bonding in biological molecules and in ice [21, 22], and analyzed the momentum dependence of vibrational states in very small volumes [23-25]. Doing them justice by providing fuller descriptions is outside the scope of this abstract, but I will try to convey how exciting these developments are in my talk at the meeting. I will also touch upon the major topic electron microscopy needs to address in its 'corrected state': minimizing radiation damage by making the best possible use of the available signals, via efficient detectors and optimized processing algorithms. 
In summary, the quest for better (S)TEM resolution via aberration correction, which was at one time dismissed as hopeless, has led to spectacular advances in both electron microscope imaging and electron energy-loss spectroscopy, and the advances show no sign of slowing down. It has been a great pleasure for me to work in this field and to participate in several of the key moments. I am particularly grateful to those who have joined me on major parts of the journey, especially to Niklas Dellby and others working at Nion, as described in reference [26], which gives my personal perspective on the key developments.

\section{References}

[1] O. Scherzer (1936) Zeit. Phys. 101, 593.

[2] O. Scherzer (1947) Optik 2, 114.

[3] J. Zach and M. Haider (1995) Optik 99, 112.

[4] O.L. Krivanek et al. (1997) Inst. Phys. Conf. Ser. 153, 35.

[5] M. Haider et al. (1998) Nature 392, 768.

[6] P.E. Batson et al. (2002) Nature 418, 617.

[7] P.W. Hawkes (2015) Ultramicroscopy 156, A1.

[8] O.L. Krivanek (1979) Scripta Chimica 14, 213.

[9] H. Rose (2008) Advances in Imaging and Electron Physics 153, 3.

[10] Y. Jiang et al. (2018) Nature 559, 343.

[11] H. Ibach (1991) Electron Energy Loss Spectrometers, Springer.

[12] O.L. Krivanek et al. (2019) Ultramicroscopy 203, 60.

[13] O.L. Krivanek et al. (2009) Phil. Trans. R. Soc. A 367, 3683.

[14] O.L. Krivanek et al. (2014) Nature 514, 209.

[15] T. Miyata et al. (2014) Microscopy 63, 377.

[16] J.A. Hachtel et al. (2019) Science 363, 2019.

[17] F.S. Hage et al. (2020) Science 367, 1124.

[18] K. Venkatraman et al. (2019) Nature Physics 15, 1237-1241.

[19] J.-C. Idrobo et al. (2018) Phys. Rev. Lett. 120, 095901.

[20] M.J. Lagos and P.E. Batson (2018) Nano Lett. 18, 4556.

[21] P. Rez et al. (2016) Nature Coms 7, article \#10945.

[22] J.R. Jokisaari et al. (2018) Adv. Mater. 30, 1802702.

[23] F.S. Hage et al. (2018) Sci. Adv. 4, 7495.

[24] R. Senga et al. (2019) Nature 573, 247.

[25] B. Plotkin-Swing et al. (2020) Ultramicroscopy 217, 113067.

[26] http://kavliprize.org/sites/default/files/KP2020nano_krivanek_autobiography.pdf 


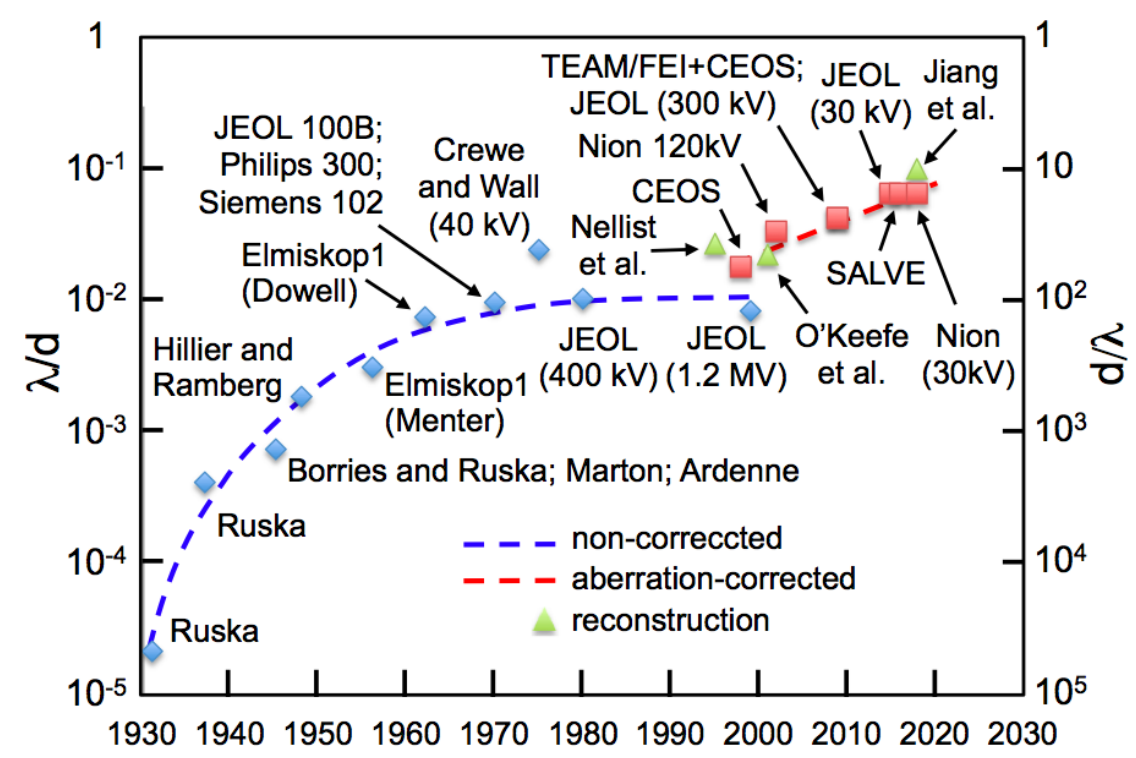

Fig. 1. Progress in the spatial resolution reached by (scanning) transmission electron microscopes ((S)TEMs), shown as the evolution of a figure of merit equal to $\lambda / \mathrm{d}$. Blue diamonds $=$ non-corrected (S)TEMs, red squares $=$ aberration-corrected (S)TEMs, green triangles $=$ computer processed.

Fig. 2. Progress in the energy resolution reached by (S)TEM electron energy loss spectrometers (EELS), shown as the evolution of a figure of merit equal to $1 /(\delta \mathrm{E} \lambda)$. Blue diamonds $=$ nonmonochromated EELS, red squares $=$ monochromated EELS.

\begin{tabular}{|r|r|r|r|r|r|r|}
\hline & \multirow{5}{*}{$k V$} & \multicolumn{5}{|c|}{ FOM $(1 /(\delta E \lambda))$} \\
\cline { 3 - 7 } & 0.1 & 0.3 & 1 & 3.4 & 10 \\
\hline 10 & 0.122 & 82.0 & 27.3 & 8.2 & 2.4 & 0.8 \\
\hline 20 & 0.086 & 116.5 & 38.8 & 11.6 & 3.4 & 1.2 \\
\hline 30 & 0.070 & 143.3 & 47.8 & 14.3 & 4.2 & 1.4 \\
\hline 60 & 0.049 & 205.6 & 68.5 & 20.6 & 6.0 & 2.1 \\
\hline 100 & 0.037 & 270.3 & 90.1 & 27.0 & 7.9 & 2.7 \\
\hline 200 & 0.025 & 398.9 & 133.0 & 39.9 & 11.7 & 4.0 \\
\hline 300 & 0.020 & 508.1 & 169.4 & 50.8 & 14.9 & 5.1 \\
\hline
\end{tabular}

Table 1. Attainable energy resolution (regular black script, values in meV) as a function of primary voltage $\mathrm{V}_{\mathrm{o}}$ and figure-of-merit $=$ $1 /(\delta \mathrm{E} \lambda)$ (blue italic script). 


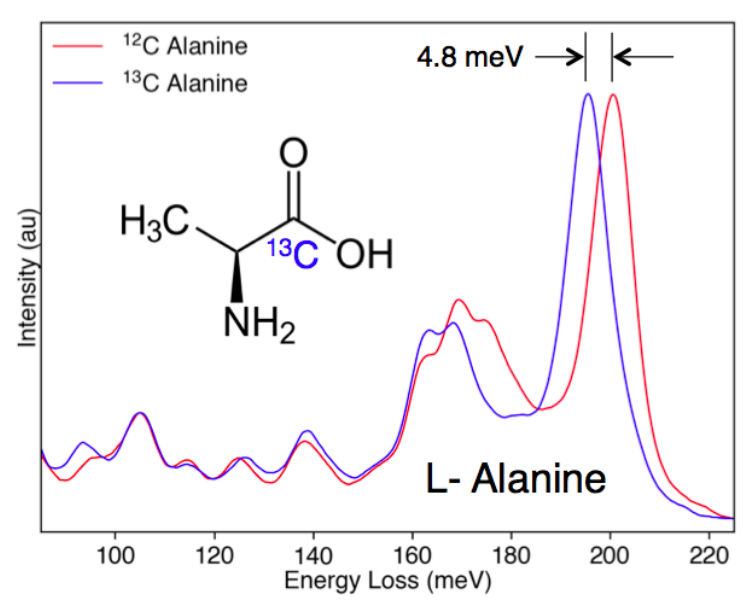

Fig. 3. Experimental energy loss spectra of L-Alanine showing the change due to substituting ${ }^{12} \mathrm{C}$ by ${ }^{13} \mathrm{C}$ at the marked location in the biological molecule. L-Alanine is an essential amino acid and the ability to map its location using of an isotopic marker should be useful for tracing biological pathways. Nion HERMES monochromated STEMEELS, $30 \mathrm{kV}$ primary voltage. Courtesy Jordan Hachtel et al., ORNL [16].


Fig. 4. Comparison of theoretically predicted vibrational spectra at different distances from a Si impurity in graphene (middle panel) with experiment (right panel). The left panel shows the experimental set-up, designed for preferentially collecting the non-dipole vibrational signal that gives good spatial localization. Nion HERMES, 60 kV. Courtesy Frederik Hage and Quentin Ramasse, Daresbury SuperSTEM [17]. 